\title{
Recognition Effect Evaluation of Radar Signals Based on Fuzzy Comprehensive Evaluation
}

\author{
Jun Han ${ }^{1, *}$, Yichun Pan ${ }^{1}$, Bo-lin Cheng ${ }^{1}$ and jing Wang ${ }^{2}$ \\ ${ }^{1}$ Information Counter Department, Air Force Early Warning Academy, Wuhan 430019, China \\ ${ }^{2}$ Beijing Yunxingyu Technology Service co., Lt, Beijing 100078, China \\ *Corresponding author
}

\begin{abstract}
The recognition of radar emitter signals (RES) is one of the key technologies in electronic countermeasures intelligence system, it's a brand new subject to scientifically evaluate the recognition effect. In this paper fuzzy comprehensive evaluation method is introduced to build relevant evaluation model for the effect evaluation, computer simulation is done to evaluate three recognition methods at last. Simulation result validates that the evaluation model is effective and has certain application prospect.
\end{abstract}

Keywords-radar emitter recognition; evaluation index; measurement of recognition rate; fuzzy comprehensive evaluation; evaluation

\section{INTRODUCTION}

Radar emitter signal recognition (RES) is a radar surveillance system against the key technology, which is a measure of the level of sophistication of radar against an important symbol of the reconnaissance system. With the improvement of the status of military radar emitter signal recognition, comprehensive and accurate identification of radar emitter signals assess the effect of providing objective for subsequent processing, scientific point of reference is needed to solve important problems. In the past, good or bad evaluation of the radar signal recognition results are based on the recognition accuracy[1-3] this single indicator, with the electromagnetic environment has become increasingly complex and diversified development of operational requirement, using a single indicator to evaluate the effectiveness of a good recognition bad clearly unscientific.

Fuzzy comprehensive evaluation method can be used for comprehensive evaluation of fuzzy things, it has been widely used in industrial and agricultural production, weather forecast, teaching quality assessment, etc[4-5]. In this article, using the mathematical statistics knowledge design experiment combined with fuzzy comprehensive evaluation method of the radar signal recognition effect for scientific evaluation.

\section{ESTABLISHING EVALUATION INDICATORS OF RECOGNITION EFFECT}

Select the evaluation indicators by our need for recognition results to determine. Starting from the performance of key concern, establish quantifiable indicators. Fuzzy comprehensive evaluation factor set elements consist of the evaluation indicators.

\section{A. The Meaning of The Recognition Rate and Calculate}

Comparison of radar signal recognition in the past is good or bad, are generally calculating recognition rate, which after many experiments, the correct identification number is divided by the total number of experiments under the conditions of the recognition rate signal, it can also be understood that ability of the method to identify the target signal under this condition. This understanding is a premise that correctly identify is " 1 ", an error or is not recognized is " 0 ".If a recognition method of identification of frankness value is $p$, then the I-TH to identify the correct probability is $p$. I-TH correctly identified obey $0-1$ distribution, the signal recognition for $\mathrm{n}$ times Bernoulli experiment, then the number of correctly identified obey $B(n, p)$ distribution, where $\mathrm{n}$ is the number of experiments.

\section{B. Proposed Recognition Rate Test Results}

From the general sense, we solve the recognition rate results are finite, it can only infinitely close to the true value but impossible to achieve true value. If we carry out different tests that can be found recognition rate is volatile, it is a variable, range changes, the way and the number of large changes can be reflected in the performance of identification.

Solving the recognition rate of conventional identification system to verify the ability of the method has some drawbacks, mainly in the following aspects:

(1) Original number of tests required to get recognition rate true value of identification system

For solving the different identification systems as well as under different external conditions, how to determine the number of tests required, the traditional method of solving the recognition rate can't be solved.

(2) The confidence interval of the recognition rate

Apparently the result of solving the traditional recognition rate is a result of the recognition test, and how to determine its degree of confidence and confidence intervals, it is unable to give.

(3) Meaning of recognition results

The traditional method for solving just identification method of test results at a time, and its capacity is equivalent to recognition method is unscientific. 
(4) The stability of recognition results

Obviously SNR interference of external factors can cause changes in the recognition rate, and how to identify the stability of scientific evaluation method, traditional methods can't do anything.

To solve the above problems, introduce the concept of identification test results[6] (MRR), the test results of $n$ were divided into $m$ groups, each of the $n / m$ test results for each group averaging, you can get $m$ MRR samples . For radar emitter signal recognition, it is assumed that there are $l$ radar emitter, then eventually generate $l \times m_{\text {MRR samples, due to }}$ the algorithms and solution consistency, just a single sample study for MRR radar emitters, namely $m$ MRR samples. Since the MRR is a variable with a distribution, mean and variance, as well as independence and external conditions, the use of which may be strictly accurate estimation of radar emitter signal recognition results.

\section{a. Prove MRR is normally distributed}

MRR for each sample, which contains $n / m$ recognition results from the above recognition result obey $0-1$ distribution. Based on the central limit theorem below prove MRR normally distributed.

Order $M=n / m$, the $M$ test results can be viewed as $M$ random variables, $x_{i}, i=1,2, \cdots, M$. Clearly, $x_{i}, i=1,2, \cdots, M$ is independent, and obey the same distribution. $\xi_{M}=\sum_{i=1}^{M} x_{i}$, according to the central limit theorem of independent and identically distributed

$$
\begin{array}{r}
\lim _{M \rightarrow \infty}\left\{\frac{\xi_{M}-M p}{\sqrt{M p(1-p)}} \leq x\right\}=\lim _{M \rightarrow \infty}\left\{\frac{\frac{1}{M} \sum_{i=1}^{M} x_{i}-p}{\sqrt{p(1-p) / M}} \leq x\right\} \\
=\int_{-\infty}^{x} \frac{1}{\sqrt{2 \pi}} \mathrm{e}^{-t^{2} / 2} \mathrm{~d} t
\end{array}
$$

Expression (1) indicates that the limit distribution of $\xi_{M}$ and $\xi_{M} / M$ is normal distribution, that is, when the test more frequently, MRR obey normally distributed. The literature[7] that the use of independent and identically distributed in the central limit theorem, $M$ is greater than 50 to meet the capacity of the big sample.

\section{b. Generate the sample of MRR}

When satisfied $M>50$, MRR is approximately obey normally distributed. With the test of the effectiveness of the following functions[8] to solve the number $M$ of tests required to generate a MRR, order $p_{0}$ is true value of the system's recognition rate, study a more rigorous study of the test:

$$
H_{0}: p=p_{0} ; \quad H_{1}: p \neq p_{0}
$$

Take the test statistic is $\sqrt{M} \frac{\bar{X}-p_{0}}{\sigma}, \sigma^{2}$ is variance of solving the system to recognize the results mean, $\bar{X}$ is the sample mean, it's theoretical value:

$$
\sigma^{2}=p_{0}\left(1-p_{0}\right) / M
$$

At this rejection region

$$
W_{1}=\left\{\left(x_{1}, x_{2}, \cdots, x_{M}\right):\left|\sqrt{M} \frac{\bar{x}-p_{0}}{\sigma}\right|>u_{1-\alpha / 2}\right\}
$$

Expression (4), $\quad \bar{x}=\frac{1}{M} \sum_{i=1}^{M} x_{i}$ is the observation value of sample mean, $x_{i}$ is the result of each test. $\alpha$ is given significance level. The effectiveness of the inspection function:

$$
\begin{aligned}
\beta(\bar{x}) & =p\left\{\sqrt{M} \frac{\bar{x}-p_{0}}{\sigma}<-u_{1-\alpha / 2} \text { 或 } \sqrt{M} \frac{\bar{x}-p_{0}}{\sigma}>u_{1-\alpha / 2}\right\} \\
& =1-p\left\{-u_{1-\alpha / 2}<\sqrt{M} \frac{\bar{x}-p_{0}}{\sigma}<u_{1-\alpha / 2}\right\}
\end{aligned}
$$

The second type of risk

$$
\begin{aligned}
1-\beta(\bar{x})= & \phi\left[u_{1-\alpha / 2}-\sqrt{M} \frac{p-p_{0}}{\sigma}\right] \\
& -\phi\left[-u_{1-\alpha / 2}-\sqrt{M} \frac{p-p_{0}}{\sigma}\right]
\end{aligned}
$$

Set the level of significance $\alpha=0.1$. Requirements when $\left(p-p_{0}\right)$ not less than 0.016 , the second type of risk is less than 0.1 , the minimum sample capacity $M$ to obey

$$
\phi\left[u_{0.95}-\frac{0.016 M}{\sqrt{p_{0}\left(1-p_{0}\right)}}\right]+\phi\left[u_{0.95}+\frac{0.016 M}{\sqrt{p_{0}\left(1-p_{0}\right)}}\right]<1.1
$$

Expression (7) can be given a minimum value of $M$, from the calculation results, $M$ not more than 95 , where select $M=100$

To generate MRR sample, also to determine the number $m$ of MRR. Depending on the test requirements, may determine the minimum values of $m$. MRR generated in the following way: 


$$
M R R_{m}=\frac{1}{100} \sum_{k=100(m-1)+1}^{100 m} x_{k}
$$

In the expression (8), ${ }_{k}$ is the result of each test, $k=1,2, \cdots, 100 m$

\section{Select Evaluation Indicators}

(1) Identifying indicators of correctness, Select the recognition rate test results (MRR) Mean.

(2) Identifying indicators of robustness, select MRR variance.

(3) On the cost aspect of indicators, Choose the time of identify.

\section{ReCognition Performance EVAluation Model BASED ON FUZZY COMPREHENSIVE EVALUATION}

According to the above discussion, construct fuzzy comprehensive evaluation model.

\section{A. Determine Factor Set}

From 2.3 evaluation indicators, determine factor set is \{MRR mean, MRR variance, recognition time\}, referred to as $U=\left\{u_{1}, u_{2}, u_{3}\right\}$.

\section{B. Choose Evaluation Set}

Choosing evaluation set is \{Good, moderate, poor\}, referred to as $V=\left(v_{1}, v_{2}, v_{3}\right)$.

\section{Factor Set Element Weight Distribution Rights}

Weight set of selection is related to assessment Background, proportional to the degree of importance of each factor with the share of the entire index system, given the weight set is $A=\left(a_{1}, a_{2}, a_{3}\right)$, and $\sum_{i=1}^{3} a_{i}=1$.

\section{For The Evaluation To Determine The Corresponding Factor Change Interval}

From the test results need to identify a single factor evaluation matrix, each of these factors will determine the variation range of reviews, as shown in Table 1, Determine these values is related to the given requirements.

TABLE I. EACH FACTOR CORRESPONDING CHANGE INTERVAL COMMENT

\begin{tabular}{|c|c|c|c|}
\hline Factor & Good & Moderate & Poor \\
\hline MRR mean & $\geq P_{1}$ & {$\left[P_{2}, P_{1}\right)$} & $\leq P_{2}$ \\
\hline MRR variance & $\leq D_{2}$ & {$\left[D_{1}, D_{2}\right)$} & $\geq D_{2}$ \\
\hline Recognition time & $\leq T_{1}$ & {$\left[T_{1}, T_{2}\right)$} & $\geq T_{2}$ \\
\hline
\end{tabular}

\section{E. Get Single Factor Evaluation Matrix}

Assuming that each evaluation indicator get $m$ values from experiments on factor set, $m$ obey sample capacity requirements. MRR mean $\geq P_{1}$ is provided, there are $m_{11}$ groups, There are $m_{12}$ groups falls on the interval $\left[P_{2}, P_{1}\right)$, MRR mean $\leq P_{2}$ is provided, there are $m_{13}$ groups, The use of fuzzy mathematics referendum law thought, MRR mean for the good of the membership is $\frac{m_{1}}{m}$,the moderate degree of membership is $\frac{m_{2}}{m}$, the poor of membership is $\frac{m_{3}}{m}$.On MRR mean that single factor evaluation results:

$$
\left[m_{11} / m, m_{12} / m, m_{13} / m\right]=\frac{1}{m}\left[m_{11}, m_{12}, m_{13}\right]
$$

Similarly, the single factor evaluation matrix:

$$
\frac{1}{m}\left[\begin{array}{lll}
m_{11} & m_{12} & m_{13} \\
m_{21} & m_{22} & m_{23} \\
m_{31} & m_{32} & m_{33}
\end{array}\right]
$$

Order $b_{i j}=\frac{m_{i j}}{m}, i, j=1,2,3, i, j=1,2,3, B$ is a single factor evaluation matrix.

\section{F. Get Fuzzy Evaluation Results}

In fact, $A$ is a fuzzy set on $U$, We want to get the results of the evaluation matrix $C$ is a fuzzy set on $V$, by fuzzy transformation[9] $A_{\text {can be mapped to }} V$ get $C$ :

$$
A \circ B=C
$$

Among them

$$
c_{j}=c_{j}=\stackrel{3}{\vee} \underset{i=1}{\vee}\left(a_{i} \wedge b_{i j}\right), j=1,2,3
$$

\section{THE SIMULATION}

\section{A. Evaluation Objects}

Take six typical radar emitter signal simulation, signal type and operating parameters as described in Table 2 below:

Where, in the frequency diversity signals of two frequency is $24 \mathrm{MHz}$ and $36 \mathrm{MHz}$,two frequency encoded signal frequency is $25 \mathrm{MHz}$ and $35 \mathrm{MHz}$, the center frequency of the remaining signal is $30 \mathrm{MHz}$, the pulse width is $10^{\mu \mathrm{S}}$, the sampling 
frequency is $120 \mathrm{MHz}$. The bandwidth of the chirp signal is $5 \mathrm{MHz}$; coding law of frequency encoded signal is [122112]; the phase-encoding rule of two-phase encoded signal is $[11,100,010,010]$; the phase-encoding rule of four-phase encoded signal is [01230312223300112012].Each signal generator 300 samples, 100 for the training, and the remaining 200 for testing.

TABLE II. EMITTER SIGNAL TYPE AND OPERATING PARAMETERS

\begin{tabular}{|c|c|c|c|c|}
\hline Number & Signal type & f/MHz & $\tau, \mu \mathrm{S}$ & PRI $/ \mu \mathrm{S}$ \\
\hline 1 & $\begin{array}{c}\text { Single carrier } \\
\text { frequency }\end{array}$ & $\begin{array}{c}400 \sim \\
2300\end{array}$ & $2 \sim 13$ & $40 \sim 800$ \\
\hline 2 & LFM & & \\
\cline { 1 - 2 } 3 & Frequency diversity & & & \\
\hline 4 & Frequency coding & & & \\
\hline 5 & Two-phase coding & & & \\
\hline 6 & Four-phase coding & & & \\
\hline
\end{tabular}

Now it takes three kinds of identification methods to evaluate the experiment. The first identification method using carrier frequency, pulse width and pulse repetition period of five conventional parameter as the three-dimensional feature vector [ $f_{R F}, \tau, P R I$ ],classifier select probabilistic neural network(PNN); The second identification method up to six signals three wavelet packet decomposition, select the class in the aggregation and separation between classes are the best ${ }^{W_{p t 6}}, W_{p t 7}[10]$ as the characteristic parameter and carrier frequency constitute a three-dimensional feature vectors[ $f_{R F}, W_{p t 6}, W_{p t 7}$ ],classifier select probabilistic neural network(PNN); The third method of selecting the same feature vector $\left[f_{R F}, W_{p t 6}, W_{p t 7}\right]$,classifier select support vector machine SVM, choose RBF kernel function.

\section{B. Establish Evaluation Model Parameters}

(1)Establish the comment corresponding factors change interval

According to the actual application requirements determined, under the test conditions, each corresponding change section reviews factors as shown in Table 3.In the Table, the recognition time only includes data processing and recognition algorithm running time, so the recognition time much shorter than the actual equipment.

TABLE III. EACH FACTORS CORRESPONDING VARIATION RANGE OF REVIEWS

\begin{tabular}{|c|c|c|c|}
\hline Factors & Good & Moderate & Poor \\
\hline MMR mean & $\geq 0.875$ & {$[0.785,0.875]$} & $\leq 0.785$ \\
\hline $\begin{array}{c}\text { MRR } \\
\text { variance } \times 10^{-3}\end{array}$ & $\leq 0.65$ & {$[0.65,0.95]$} & $\geq 0.95$ \\
\hline $\begin{array}{c}\text { Identification } \\
\text { time/s }\end{array}$ & $\leq 0.25$ & {$[0.25,0.34]$} & $\geq 0.34$ \\
\hline
\end{tabular}

(2) Establish weight

When the weight of each factor changes, the composite score values will change, which means that the results of the comprehensive evaluation is dynamic, with the actual signal environment and application requirements change, which is currently operational requirements coincide. According to specific needs determine the weight vector is $(0.5,0.3,0.2)$.

\section{(3) Fuzzy comprehensive evaluation}

Use optional radar signal data and identification methods for the identification of three experiments, each method for 2000 times independently recognition experiment, per 100 recognition results determine a MRR sample, each method a total of 20 MRR samples. Final evaluation results got by the model shown in Table 4:

TABLE IV. ASSESSMENT RESULTS OF THE THREE IDENTIFICATION METHODS

\begin{tabular}{|c|c|c|c|}
\hline Comment & Good & Moderate & Poor \\
\hline Recognition method 1 & 0.105 & 0.59 & 0.305 \\
\hline Recognition method 2 & 0.545 & 0.38 & 0.075 \\
\hline Recognition method 3 & 0.62 & 0.105 & 0.275 \\
\hline
\end{tabular}

Assessment conclusion: in the case of this test, the best performance is method 3 , method 1 is worst, method 2 is center. Theoretical analysis, factor weights vector is $(0.5,0.3,0.2)$, it means that this test requires the identification correctness highest, followed by stability and recognition time. method 1 compared to method 2,method 2 using signal wavelet packet coefficients as the characteristic parameter, improve the recognition accuracy of the signal; Compared to method 3 and method 2, the classifier select SVM, it solve the small sample, the high-dimensional pattern recognition problem more advantages than neural networks, but large amount of computation at the expense of time.

\section{CONCLUSION}

Assess radar emitter recognition results have important applications significance, traditional evaluation methods to identify good or bad based on a single indicator and a small number of experiments to test the capacity, the lack of scientific. Based on the test results of recognition rate (MRR), the tool will use fuzzy comprehensive evaluation method to assess the effect of radar emitter identification, evaluation model established by computer simulation of the three identification methods were evaluated, the simulation results show the assessment of the feasibility of the method has certain reference value.

\section{REFERENCES}

[1] Jun Han, Ming-hao He, Yan Mao, Ming-qiu Ren. A New Method for Recognizing Radar Radiating-source [J]. ICWAPR'2007, 4:1665-1668.

[2] Zhang G X, Hu L Z, Jin W D. Quantum Computing Based Machine Learning Method and Its Application in Radar Emitter Signal Recognition [J]. Lecture Notes in Artificial Intelligence, 2004, 3131, 92-103.

[3] Zhang Guo-zhu. Research on Radar Emitter Recognition technology[D].Changsha: National University of Defense Technology, 2005.

[4] He Zhong-xiong. Mathematical Physics[M]. Tianjin: Tianjin Science and Technology Publishing House, 1982. 188 218. 
[5] Li Hong-xing. The Fuzzy Mathematics Method and its Application[M]. Tianjin: Tianjin Science and Technology Publishing House, 1993. $132 \sim 136$.

[6] Zhuang Zhao-wen. Automatic Target Recognition Effect Evaluation Technology[M]. Beijing: National Defence Industry Press, 2006. 61 62.

[7] Sheng Zhou. Probability and Mathematical Statistics[M]. Beijing: Higher Education Press, 1989.

[8] Yu Geng. Advanced Engineering Mathematics[M]. Wuhan: Huazhong University of Science Press, 1995.

[9] Zhang G X. Research on Radar Emitter Signal Intelligent Recognition Method[D], Chengdu: Southwest Jiaotong University, 2005. 